\title{
Assessing age in the desert tortoise Gopherus agassizii: testing skeletochronology with individuals of known age
}

\author{
Amanda J. Curtin ${ }^{1,4}$, George R. Zug ${ }^{2, *}$, Philip A. Medica ${ }^{3}$, James R. Spotila ${ }^{1}$ \\ ${ }^{1}$ Department of Bioscience and Biotechnology, Drexel University, 3141 Chestnut St., Pennsylvania 19104, USA \\ ${ }^{2}$ Department of Vertebrate Zoology, National Museum of Natural History, Smithsonian Institution, PO Box 37012 , \\ Washington, DC 20013-7012, USA \\ ${ }^{3}$ USGS-Biological Resources Division, Western Ecological Science Center, 160 N. Stephanie St., Henderson, \\ Nevada 89074, USA
}

${ }^{4}$ Present address: Academic Resource Center, Duke University, PO Box 90694, Durham, North Carolina 27708, USA

\begin{abstract}
Eight desert tortoises Gopherus agassizii from a long-term mark-recapture study in the Mojave Desert, Nevada, USA, afforded an opportunity to examine the accuracy of skeletochronological age estimation on tortoises from a seasonal, yet environmentally erratic environment. These 8 tortoises were marked as hatchlings or within the first $2 \mathrm{yr}$ of life, and their carcasses were salvaged from predator kills. Using a blind protocol, 2 skeletochronological protocols (correction-factor and ranking) provided age estimates for a set of 4 bony elements (humerus, scapula, femur, ilium) from these tortoises of known age. The age at death of the tortoises ranged from 15 to $50 \mathrm{yr}$. The most accurate protocol - ranking using the growth layers within each of the 4 elements - provided estimates from 21 to $47 \mathrm{yr}$, with the highest accuracy from the ilia. The results indicate that skeletochronological age estimation provides a reasonably accurate method for assessing the age at death of desert tortoises and, if used with a large sample of individuals, will provide a valuable tool for examining agerelated mortality parameters in desert tortoise and likely in other gopher tortoises (Gopherus).
\end{abstract}

KEY WORDS: Age - Sexual maturity - Skeletochronology - Testudines - Mojave Desert · North America Resale or republication not permitted without written consent of the publisher

\section{INTRODUCTION}

Age is an essential datum for demographic studies, constructing life tables, and deciphering life histories (Slobodkin 1963, p. 34). For many animals, gathering age data on individuals is a relatively easy task because of their visibility, rapid growth, and short life spans. Age determination becomes increasingly difficult with long-lived animals, especially for ones that disappear from researchers' observations for lengthy periods of time each year. Turtles and tortoises are in this latter category (Congdon \& Gibbons 1990). Desert tortoises often limit their foraging to a few months each year (Averill-Murray et al. 2002), owing to the harshness of their environment and the necessity to escape the intense heat and dehydrating conditions on the surface of the desert floor. Whether difficult to obtain or not, age estimates for individuals of multiple populations of the desert tortoise are critical and necessary for conservation management of this threatened species (Fish \& Wildlife Service 1994, Tracy et al. 2004). Skeletochronological age estimates have proved useful in modeling sea turtle populations (Heppell et al. 1999) and will broaden the age database for desert tortoises.

\section{Desert tortoise Gopherus agassizii}

Four species of tortoise occur in North America. All 4 are members of the genus Gopherus (Ernst et al. 1994), and all 4 live in dry habitats, with aridity and environ- 
mental harshness increasing from east to west. The bolson tortoise Gopherus flavomarginatus (Chihuahuan Desert) and the desert tortoise G. agassizii (Sonoran and Mojave Deserts) live in truly arid environments. In addition to enduring the physiological stressfulness of its environment, G. agassizii is nearly exclusively herbivorous and largely energy-dependent on annual plants and perennial grasses that appear with seasonal rains and disappear rapidly thereafter (Nagy \& Medica 1986). For the desert tortoise, growth is seasonal, associated with seasonal rainfall and the appearance of annual plants (Medica et al. 1975, Germano 1994). This dependence results in relatively slow growth, with maturity occurring at a minimum of $15 \mathrm{yr}$, later for most individuals (Germano 1994).

This maturity estimate is, however, a species-wide one. Climate is significantly different between the Sonoran and Mojave Deserts, the former with summer and winter rains followed by fall and spring droughts and the latter with primarily winter rains and a lengthy dry season, followed by isolated, localized summer thunderstorms. Additionally, microenvironments within each of the deserts result in different dietary regimes (Oftedal 2002); hence, different growth rates and ages of maturity are expected. Although markrecapture studies are the most accurate method of aging individuals in reptilian populations (Zug 1991), other less time-demanding methodologies are required. Skeletochronology is one such technique, and its accuracy can be assessed and its results calibrated through the use of known-aged individuals.

\section{Age estimations}

Previous estimates of age and growth rates in desert tortoises have used scute laminae counts, annual body size measurements, and body mass (Medica et al. 1975, Turner et al. 1987, Germano 1988, 1994). Germano's 1988 study showed that the number of scute laminae more or less corresponded to the ages of semicaptive tortoises and were within 1 to 2 scute laminae less than the known-aged tortoises in wild desert tortoises of Rock Valley. He proposed that both the number of scute laminae and long-bone growth layers could be used owing to the high correlation ( $\mathrm{r}=0.92)$ between them, and that these age estimates could be used to age juveniles and subadults to about $25 \mathrm{yr}$ of age. Subsequently, after studying the formation of scute laminae in juvenile desert tortoises raised in captivity, Tracy \& Tracy (1995) argued that Germano's 1998 study did not reflect age accurately. However, the artificiality of the rearing conditions in the Tracy \& Tracy (1995) study does not support their premise that scute laminae are invalid estimators of age, although their study does demonstrate the strong influence of environmental factors on scute growth. More recently, a study of scute laminae formation in juveniles from the Mojave and Colorado Deserts (Berry 2002) found that laminae counts varied by desert region. Berry's data from 11 localities showed that a variable number (0 to 3 ) of laminae are produced each year and, further, that at different sites (different environments), tortoises produce laminae at different rates. Nevertheless, she stated: 'in most cases the modes and medians show 1.0 ring [lamina] formed per year,' although she identified the necessity of determining the average lamina-formation rate for each site. Berry also noted that the observation of multiple scute laminae for a single year was also derived in part from small samples and/or short-duration studies. Even though the use of annual growth layers remains contentious for some turtle biologists, a recent literature review of growth and aging (Wilson et al. 2003) demonstrated that laminae number is an accurate estimator age for many turtle species and populations until individuals therein attain maturity. That review also demonstrated the need to verify the $1 \mathrm{yr}=1$ lamina hypothesis for each species.

The question remains, how do we obtain accurate age estimates without long-term mark-recapture studies? There are no published or validated assessments of age structure in desert tortoises. The markrecapture project in Rock Valley (three 9 ha fenced sites) documents a cohort of known-aged individuals, but it is not a sample that reflects the entire population of Rock Valley area. Thus, age structure data for most desert tortoise populations are anecdotal at best and have been based primarily upon body sizes. If we are to discern and compare life history evolution among desert tortoise populations, we need a method to estimate age accurately and quickly. Skeletochronology has been shown to be an accurate aging method for many reptiles and is the best method for estimating age structure when mark-recapture data are not available (Castanet \& Smirina 1990). Our goal is to test the validity of the $1 \mathrm{yr}=1$ lamina hypothesis for the desert tortoise and to determine which salvageable skeletal element yields the most accurate estimates.

\section{MATERIALS AND METHODS}

Mojave sample. In 1963, a desert tortoise markrecapture study was begun in Rock Valley, about $110 \mathrm{~km}$ northwest of Las Vegas, Nevada (Medica et al. 1975); it continues to the present. One of the initial goals was to document incremental growth of a cohort of hatchling and very small tortoises. Tortoises between 49 and $74 \mathrm{~mm}$ plastron length at first capture 
were marked between 1963 and 1966; size and scute growth layer yielded age estimates of 1 to 4 yr old for this sample (Medica et al. 1975, Turner et al. 1987). The mark-recapture data showed that growth among Rock Valley juvenile tortoises occurred nearly exclusively in the spring and early summer (Medica et al. 1975). They also determined that juvenile growth averaged about $9 \mathrm{~mm} \mathrm{yr}^{-1}$ and was correlated positively with winter rainfall and the production of winter annual plants.

Our skeletochronological sample consists of 8 of these Rock Valley marked individuals found dead in subsequent surveys between 1996 and 2003, specifically 6 adult females (USNM 560933, 560934, 560935, 560936, 560939, 560941, see Table 1) and 2 adult males (USNM 560937, 560938) of known age. An additional sample of 13 juvenile tortoise carcasses from Piute Valley, southern Nevada (6 from the Christmas Tree Permanent Study Plot and 7 from approx. $10 \mathrm{~km}$ south of this plot) were used for the calculation of an estimate of relative periosteal growth rate, the correction factor (CF). CF is a value used to estimate the number of periosteal growth layers lost through resorption in the center of the growing humerus. Standard shell measurements (carapace length and width, CL and CW, plastron length, PL) were recorded from intact shells and, where possible, longitudinal length (LL), proximal width (PW), midshaft width (MW), and distal width (DW) from all long bones was also recorded. At the microscopic level, we recorded a series of measurements in addition to counting the number of visible periosteal growth layers for each bony element: diameter of the resorption core $(\mathrm{rcD})$; successive diameters of periosteal layers $\left(\mathrm{plD}_{\mathrm{n}}\right)$; and outside diameter of bone. These measurements were made with a stage micrometer perpendicular to the longitudinal axis of the element and along the anterior-posterior axis of the bone. The successive $\mathrm{plD}_{\mathrm{n}}$ measurements represent the diameter of the bone element at the beginning of each growth period and, hence, a record of the size of the tortoise at the beginning (or end) of each growth interval. Although not used herein, the strong correlation between the diameters of the bone elements and CL or PL permits the estimation of shell length at successive growth intervals via a back-calculation procedure using a regression equation.

Skeletochronological methodology. Initially, to identify those skeletal elements with the best growthlayer signatures, we sectioned all elements of the appendicular skeleton, cervical and dorsal vertebrae, carapace elements (nuchal, neurals, peripherals), and a plastron element (epiplastron). The girdle and limb bones, specifically the humerus, femur, scapula, and ilium, had the most and clearest growth layering.

Skeletochronological examination requires histological preparation. We fixed, decalcified and processed bones with standard histological protocols. All bones embedded in Paraplast Plus@ $\odot$ were cross-sectioned $20 \mu \mathrm{m}$ at midshaft of each bone using a rotary microtome and stained with hematoxylin and eosin. We measured all growth layer diameters along the dorsoventral plane using a transmitting light microscope.

We use 2 skeletochronological methods to estimate ages: the ranking protocol (RP) described by Zug (1990, 1991) and the CF method described by Parham \& Zug (1997). CF uses an estimate of the number of growth layers plus the actual number of layers remaining in the bony element to yield an age estimate. CF is the mean slope $(\beta)$ of successive growth diameters, determined from a sample of small tortoises (Piute Valley sample). The equation $\mathrm{rcD}-$ hatchling humerus diameter $\div \mathrm{CF}$ yields an estimate of the number of growth diameters lost. This estimate is added to the number of growth diameters observed to give an estimate of the individual's total number of diameters and an estimate of age (further details in Zug et al. 2006).

$\mathrm{RP}$ relies on the sequential arrangement of individuals by their resorption core diameters, from smallest to largest. Each row of data represents 1 individual and contains its successive growth diameters (layers) and the columns produced by addition of the successive larger resorption-cored individuals. Each column represents a growth diameter class, as well as an estimated age class (Zug et al. 2006). Two age estimates were obtained using RP. In the first, ranking was based on successive growth layers; we termed this the GL ranking protocol. An alternate method used the periosteum diameter (external or outer diameter of the bone's cross-section) to represent the final growth layer rather the last complete growth layer; we termed this the PD ranking protocol. The latter method uses the same age (or diameter) classes as GL ranking but instead assigns the visible growth layers into age classes. This modification, if GL and PD age estimates correlated, would provide a useful alternative to obtaining age estimates without sectioning bones.

Each specimen potentially has 8 age estimates, 2 (RP and $\mathrm{CF}$ ) for each of the 4 bones (humerus, scapula, femur, ilium). However, none of our known-aged tortoises underwent all 8 estimates because all specimens were salvaged and all had died through predation. The killing and eating of each tortoise had damaged the shell and some of the elements had been consumed; subsequently, scavengers added to the damage and loss of limb and girdle elements. The size, known age, and skeletal element data are summarized in Table 1. To estimate age we used a blind protocol, i.e. we made all age estimates without knowledge of the known ages of the individual specimens, to avoid a bias of the estimates. 
Table 1. Gopherus agassizii. Data summary for the known-age tortoises from Rock Valley, Nevada. All data are for the tortoises at the time of their death. Specimens are housed in the Smithsonian's National Museum of Natural History (USNM) and are assigned unique catalog numbers. CL: carapace

length; CW: carapace width; PL: plastron length; -: no data

\begin{tabular}{|lccccc|}
\hline $\begin{array}{l}\text { Specimen } \\
\text { identifier }\end{array}$ & Sex & $\begin{array}{c}\text { Age } \\
(\mathrm{yr})\end{array}$ & $\begin{array}{c}\mathrm{CL} \\
(\mathrm{mm})\end{array}$ & $\begin{array}{c}\mathrm{CW} \\
(\mathrm{mm})\end{array}$ & $\begin{array}{c}\mathrm{PL} \\
(\mathrm{mm})\end{array}$ \\
\hline 560933 & Female & 15 & 189 & 138 & 176 \\
560934 & Female & 36 & 220 & 172 & 208 \\
560935 & Female & 34 & - & 141 & 169 \\
560936 & Female & 40 & 225 & 184 & 215 \\
560937 & Male & 40 & 226 & 182 & 223 \\
560938 & Male & 50 & 254 & 185 & 233 \\
590939 & Female & 42 & 243 & 181 & 219 \\
560941 & Female & 40 & 228 & 180 & 217 \\
\hline
\end{tabular}

Statistical analyses. We assessed similarity between age estimates and known ages, but our primary goal was to determine which skeletochronological methodology yields the closest match between the known ages and the age estimates. We examined the similarity (or lack thereof) with both parametric and nonparametric statistics, using SYSTAT for Windows, version 11. Nonparametric tests include the sign test, Spearman's rank correlation coefficient $\left(\mathrm{r}_{\mathrm{S}}\right)$ and Kendall's $\tau$ rank comparisons. Recognizing the difficulties of small sample sizes and associated deviation from the assumptions of parametric statistics, we examined the difference between skeletochronology age estimates and known age using the mean squared error (MSE) and root mean squared error (RMSE) to assess which estimate and bony element yielded the least error and bias to the known ages. Further, we assessed the relationship between known age and skeletochronology age and also age (independent variable) and size (dependent variable) using the Pearson productmoment correlation coefficient and least square regressions.

\section{RESULTS}

Skeletochronology, like dendrochronology, is dependent upon the number of growth layers. Unlike the latter, skeletochronology of turtles and tortoises must contend with the resorption of earlier deposited layers. For our sample, the number of periosteal layers for all individuals was less than their known age (Table 2). An aspect of these lost layers is the difference in the amount of resorption in different bony elements (Table 2). For most tortoises, the ilium lost the fewest layers. These differences also show an association with known age, i.e. older tortoises have lost more layers.
Table 2. Gopherus agassizii. Number of periosteal layers in the different bony elements of known-age Rock Valley tortoises. CL: carapace length at death. Specimens are housed in the Smithsonian's National Museum of Natural History (USNM) and are assigned unique catalog numbers. -: no data

Specimen CL No. of visible periosteal layers Known (mm) Humerus Femur Scapula Ilium age (yr)

\begin{tabular}{ccccccc}
\hline 560933 & 189 & - & 12 & - & - & 15 \\
560934 & 220 & 18 & 22 & 27 & 28 & 36 \\
560935 & - & 20 & - & - & 20 & 34 \\
560936 & 225 & 20 & - & - & 22 & 40 \\
560937 & 226 & - & - & 19 & 21 & 40 \\
560938 & 254 & - & - & - & 33 & 50 \\
590939 & 243 & 28 & - & - & - & 42 \\
560941 & 228 & 28 & - & 17 & - & 40 \\
\end{tabular}

The inequality of bony elements in different individuals prevents a statistical test of the homogeneity of layer loss both among different bones from a single individual and in the same bone among multiple individuals.

The Piute Valley sample comprises juveniles ranging from 54 to $123 \mathrm{~mm}$ CL. Their ages were not known; subsequent estimated ages range from 3 to $17 \mathrm{yr}$. They were selected as an appropriate subset for use of the diameters of their resorption cores and early growth layers to calculate $\mathrm{CF}$. Because the earliest periosteal layers are lost first, knowledge of the earliest growth layer diameters and thicknesses are essential to avoid overestimates of age that would result from the use of the proportionately thinner growth layers in older juveniles and adults.

The age estimates show a disparate pattern at all levels of comparison. Within a single element - for example the ilium (Table 3) — the differences among the 3 age estimates of an individual range from 4 to $20 \mathrm{yr}$ $(n=5)$. Using the same bone, the difference between known age and estimates are: GL, 0 to $9 \mathrm{yr} ; \mathrm{PD}, 5$ to $23 \mathrm{yr}$; CF, 5 to $16 \mathrm{yr}$. A similar level of variation occurs within the humeral age estimates $(n=5)$. Given the even smaller sample sizes ( $\mathrm{n} \leq 3$ ), variation appears similar in the estimates calculated from the scapulae and femora.

A visual inspection of the results of the 3 types of age estimates indicates GL estimates are most similar to known age and that among all estimates the closest to known age were derived using the ilia (Table 3). Statistical tests support this impression. Sign tests show that within a bone, CF estimates consistently yield the lowest age estimates, with the probability of equal numbers of positive and negative differences from known ages, $\mathrm{p}<0.10$ for all 4 bones. GL and PD estimates tend to be somewhat more similar to one another; however, differences remain 1-sided, e.g. $p=0.375$ in GLs with the humeri, but $p=1.00$ with the ilia. GL age estimates 
Table 3. Gopherus agassizii. Age estimates obtained by different estimation protocols for different bony elements of known-age Rock Valley tortoises. GL: growth layer ranking; PD: periosteal diameter ranking; CF: correction-factor; -: no data

\begin{tabular}{|c|c|c|c|c|c|c|c|c|c|c|c|c|c|}
\hline \multirow{3}{*}{$\begin{array}{l}\text { Specimen } \\
\text { identifer }\end{array}$} & \multicolumn{12}{|c|}{ - Age estimates (yr) } & \multirow{3}{*}{$\begin{array}{l}\text { Known } \\
\text { age (yr }\end{array}$} \\
\hline & \multicolumn{3}{|c|}{ Humerus } & \multicolumn{3}{|c|}{ Scapula } & \multicolumn{3}{|c|}{ Femur } & \multicolumn{3}{|c|}{ Ilium } & \\
\hline & GL & $\mathrm{PD}$ & $\mathrm{CF}$ & GL & $\mathrm{PD}$ & $\mathrm{CF}$ & GL & $\mathrm{PD}$ & $\mathrm{CF}$ & GL & $\mathrm{PD}$ & $\mathrm{CF}$ & \\
\hline 560933 & - & - & - & - & - & - & 21 & 24 & 15 & - & - & - & 15 \\
\hline 560934 & 35 & 36 & 21 & 31 & 23 & 31 & 33 & 23 & 25 & 40 & 31 & 31 & 36 \\
\hline 560935 & 29 & 23 & 22 & - & - & - & - & - & - & 34 & 20 & 23 & 34 \\
\hline 560936 & 34 & 36 & 23 & - & - & - & - & - & - & 40 & 27 & 25 & 40 \\
\hline 560937 & - & - & - & 35 & 27 & 22 & - & - & - & 39 & 27 & 24 & 40 \\
\hline 560938 & - & - & - & - & - & - & - & - & - & 47 & 27 & 35 & 50 \\
\hline 590939 & 46 & 48 & 32 & - & - & - & - & - & - & - & - & - & 42 \\
\hline 560941 & 35 & 49 & 29 & 29 & 24 & 20 & - & - & - & - & - & - & 40 \\
\hline
\end{tabular}

are predominantly lower than the PD ones using the humeri and femora and higher in the scapulae and ilia. Spearman's $r_{S}$ and Kendall's $\tau$ permit a comparison of rank order of the estimates for each bone. Centering on the larger samples, both statistics reveal the strongest match of known to estimated ages for the ilia, i.e. $\mathrm{r}_{\mathrm{S}}=$ $1.00 \mathrm{GL}, 1.00 \mathrm{CF}$ and $\tau=1.00 \mathrm{GL}, 0.97 \mathrm{CF}$.

Even though the preceding statistics demonstrate that GL ranking most closely matches the age ranking in the known-aged tortoises, they do not offer a measure of how closely the estimates are to the actual ages of the tortoises. Do any of our protocols give estimates identical to known ages? Once again, inspection (Table 3) demonstrates that for both humeri and ilia, GL estimates are most similar to known age. The best estimates derive from the ilium, with 2 estimates identical to, 1 older than (by 4 yr) and 2 younger than (by 1 and 3 yr) known age.

Parametric statistics yield the same results. Mean differences between known and estimated ages (pooling the data for all 4 bones) shows that GL estimates differ least from known ages (Table 4). Similarly, correlation analysis reveals the highest association of GL estimates and known ages $\left(\mathrm{R}^{2}=0.877 \mathrm{GL}\right.$, $0.127 \mathrm{PD}, 0.765 \mathrm{CF}$ ), and highest association of GL estimates to body size $\left(\mathrm{CL}: \mathrm{R}^{2}=0.924\right.$ known age, $0.886 \mathrm{GL}$, 0118 PD, $0.818 \mathrm{CF}$ ).

\section{DISCUSSION}

Discrete growth layers occurred in all 4 bones: humerus, scapula, femur, and ilium. The number of visible layers was roughly equivalent among the 4 bones (Table 2), with the femur averaging the fewest and the humerus and il- ium equally the greatest (22.8 periosteal layers). This comparison, however, is biased by the failure to recover each of the 4 elements from all 8 carcasses. Both the predator and subsequent scavengers consumed or scattered skeletal elements, thus preventing the recovery of all elements from the salvaged carcasses, with the exception of 1 individual. The unequal availability of bones and the desire to provide a technique for the greatest number of salvaged carcasses (both for this study and subsequent ones) led to a survey of a variety of skeletal elements and the identification of the preceding 4 bones as the ones containing the most discrete layers. The presence of discrete layering in the humerus and femur is not surprising as these elements are the standard ones used in other skeletochronological studies of amphibians and reptiles (e.g. Castanet \& Smirina 1990, Castanet et al. 1993). The ilium and scapula have not been used previously and our discovery of their discrete periosteal layering broadens the research opportunities for others determining age in salvaged turtles. The more cylindrical morphology of the testudine scapula and ilium make

Table 4. Gopherus agassizii. Comparison of estimated ages derived from the 3 skeletochronological protocols to the known ages of tortoises from Rock Valley, Nevada. GL: growth layer ranking; PD: periosteal diameter ranking; CF: correction-factor; $\mathrm{n}$ : total number of bones tested; mean difference (in years): mean difference between known and estimated ages; MSE: mean squared error of differences; RMSE: root mean squared error; $t$ : Student's $t$-test

\begin{tabular}{|c|c|c|c|c|c|c|}
\hline $\begin{array}{l}\text { Age } \\
\text { est. } \\
\text { protocol }\end{array}$ & $\begin{array}{l}\text { Sample } \\
\text { size (n) }\end{array}$ & $\begin{array}{c}\text { Mean } \\
\text { difference } \\
\text { (SE) }\end{array}$ & $\begin{array}{l}\text { MSE } \\
\text { (SE) }\end{array}$ & $\begin{array}{l}\text { RMSE } \\
\text { (SE) }\end{array}$ & $\begin{array}{c}t \\
(14 \mathrm{df})\end{array}$ & $\mathrm{p}$ \\
\hline GL & 15 & $\begin{array}{l}-2.07 \\
(1.16)\end{array}$ & $\begin{array}{l}23.00 \\
(7.07)\end{array}$ & $\begin{array}{c}3.93 \\
(0.73)\end{array}$ & 1.787 & 0.096 \\
\hline $\mathrm{PD}$ & 15 & $\begin{array}{l}-7.60 \\
(2.51)\end{array}$ & $\begin{array}{l}145.73 \\
(33.66)\end{array}$ & $\begin{array}{l}10.80 \\
(1.44)\end{array}$ & 3.032 & 0.009 \\
\hline $\mathrm{CF}$ & 15 & $\begin{array}{c}-12.07 \\
(1.41)\end{array}$ & $\begin{array}{l}173.40 \\
(30.14)\end{array}$ & $\begin{array}{l}12.07 \\
(1.41)\end{array}$ & 8.56 & $<0.001$ \\
\hline
\end{tabular}


them more likely candidates for discrete and uniform periosteal growth layering. Additionally, their size and location within the shell increases the likelihood of their discovery in depredated turtles. We note that we view skeletochronology as a technique for age determination in salvaged testudines, and we certainly do not recommend the sacrifice of individual turtles for age determination.

The unequal sampling of bony elements potentially biases our analysis of accuracy of age estimation among the 3 methodologies (GL ranking, PL ranking, and CF estimation); however, our comparisons using a variety of nonparametric and parametric statistical tests yield the same results and conclusions. First, the most accurate age estimates (i.e. those most similar or identical to known age) for adult desert tortoises derive from the ilium, and the most accurate method is GL ranking. Where ages are over- or underestimated, it is possible that we classified a double line of arrested growth (LAG) (2 periods of arrested growth within 1 growth cycle) as 2 single LAGs or vice-versa, or that a LAG was too indistinct and not counted.

CF estimates have the greatest deviation from the known ages for adult tortoise, i.e. more than $34 \mathrm{yr}$ and $220 \mathrm{~mm}$ CL in our sample. The likely reason that CF estimates are significantly lower in adults is that this method relies solely on hatchling and juvenile growth to determine resorbed growth rings in adults. Even though cortical remodeling is considered to some degree (within resorption core diameters), it is mainly considered in regard to juvenile growth rate (in which remodeling is usually very limited) and not the rate and extent to which it occurs in adults. CF estimates are, however, the most similar to known age in the juveniles. The juvenile sample consists of only 3 age estimates; hence, it is too small to reach any firm conclusion, but it is indicative and recommends future testing.

Our study demonstrates that it is possible to obtain reliable age estimates for desert tortoises using skeletochronology where known-age, mark-recapture data are unattainable. Skeletochronology is a relatively inexpensive, non-labor intensive method that can be used across populations. Carcasses found in the wild, which were of limited benefit before, now can be used to obtain age estimates and, along with the size of the carcasses, can be used to model growth rates in localized samples without the intense commitment required by mark-recapture studies.

Acknowledgements. We gratefully acknowledge the Panaphil Foundation for a fellowship to A.J.C., assisting her predoctoral research. We thank the Smithsonian Institution's Division of Amphibians and Reptiles (USNM) for access to the skeletal material and also the Nevada Department of Wildlife also for loan of the skeletal specimens. F. B. Turner and J. R Lannom Jr. initiated field studies at Rock Valley in 1963, hence enabling this study of known-aged tortoises. A contract (DE-AC03-76-SF00012) between the US Department of Energy and the University of California and the International Biological Program/Desert Biome (National Science Foundation Grant BG 32139) supported collection between 1963 and 1980. Additionally, we thank J. Yee and J.F. Parham for their constructive criticism of our manuscript. We appreciated the assistance of field study volunteers, especially P. Greger, K. Murphy, M. Burroughs, and J. Burroughs, who facilitated data collection between 1981 and 2004. We also thank numerous other individuals from the following organizations: Bechtel Nevada Corp.; Bureau of Land Management; EG\&G Energy Measurements; Reynolds Electrical Engineering Co.; National Museum of Natural History-Smithsonian; Student Conservation Association; US Fish \& Wildlife Service; US Geological Survey/Biological Resources Division; US Geological Survey/Water Resources Division; US International Biological Program; University of California, Los Angeles; University of Nevada, Las Vegas; and University of Nevada, Reno. These organizations/individuals provided services and courtesies that aided the field work and the skeletochronological research. We also thank the US Department of EnergyNevada Operations Office for continued encouragement and support that have enabled the collection of such an irreplaceable data set. The Nevada Department of Wildlife issued a permit each year to allow the handling of tortoises and data collection. A US Fish and Wildlife Threatened Species permit TE 59747 issued to P.A.M. similarly enabled our field studies following the listing of the desert tortoise as a threatened species in 1989.

\section{LITERATURE CITED}

Averill-Murray RC, Martin BE, Bailey SJ, Wirt EB (2002) Activity and behavior of the Sonoran desert tortoise in Arizona. In: Van Devender, TR (ed) The Sonoran desert tortoise: natural history, biology, and conservation. University of Arizona Press \& Arizona-Sonora Desert Museum, Tucson, AZ, p 135-158

Berry KH (2002) Using growth ring counts to age juvenile desert tortoises (Gopherus agassizii) in the wild. Chelonian Conserv Biol 4:416-424

Castanet, J, Smirina E (1990) Introduction to the skeletochronological method in amphibians and reptiles. Ann Sci Nat Zool 11:191-196

Castanet J, Francillon-Vieillot HJ, Meunier FJ, de Ricqlès A (1993) Bone and individual ageing. In: Hall BK (ed) Bone, Vol 7. CRC Press, London, p 245-283

Congdon JD, Gibbons JW (1990) Evolution of turtle life histories. In: Gibbons JW (ed) Life history and ecology of the slider turtle. Smithsonian Institution Press, Washington, DC, p 45-56

Ernst CH, Lovich JE, Barbour RW (1994) Turtles of the United States and Canada. Smithsonian Institution Press, Washington, DC

Fish \& Wildlife Service (1994) Desert tortoise (Mojave population) recovery plan. US Fish \& Wildlife Service, Portland, OR

$>$ Germano DJ (1988) Age and growth histories of desert tortoises using scute annuli. Copeia 1988:914-920

Germano DJ (1994) Growth and age at maturity of North American tortoises in relation to regional climates. Can J Zool 72:918-931

Heppell SS, Crowder LB, Menzel TR (1999) Life table analy- 
sis of long-lived marine species with implications for conservation and management. Am Fish Soc Symp 23: 137-148

Medica PA, Bury RB, Turner FB (1975) Growth of the desert tortoise (Gopherus agassizii) in Nevada. Copeia 1975: 639-643

Nagy KA, Medica PA (1986) Physiological ecology of the desert tortoise in southern Nevada. Herpetologica 42:73-92

Oftedal OT (2002) Nutritional ecology of the desert tortoise in the Mojave and Sonoran Deserts. In: Van Devender TR (ed) The Sonoran desert tortoise: natural history, biology, and conservation. University of Arizona Press \& ArizonaSonora Desert Museum, Tucson, AZ, p 194-241

Parham JF, Zug GR (1997) Age and growth of loggerhead seaturtles (Caretta caretta) of coastal Georgia: an assessment if skeletochronological age-estimates. Bull Mar Sci 61:287-304

Slobodkin LB (1963) Growth and regulation of animal populations. Holt, Rinehart and Winston, New York

Tracy CR, Tracy CR (1995) Estimating age of desert tortoises

Editorial responsibility: Michael Mahony,

Callaghan, New South Wales, Australia
(Gopherus agassizii) from scute rings. Copeia 1995: 964-966

Tracy CR, Averill-Murray R, Boarman WI, Delehanty D and others (2004) Desert tortoises recovery plan assessment. Nevada Fish \& Wildlife Office, US Fish \& Wildlife Service, Reno, NV

Turner FB, Medica PA, Bury RB (1987) Age-size relationships of desert tortoises (Gopherus agassizii) in southern Nevada. Copeia 1987:974-977

Wilson DS, Tracy CR, Tracy CR (2003) Estimating age of turtles from growth rings: a critical evaluation of the technique. Herpetologica 59:178-194

Zug GR (1990) Age determination of long-lived reptiles: some techniques for seaturtles. Ann Sci Nat Zool 11:219-222

Zug GR (1991) Age determination in turtles. Herpetol Circ 20: $1-28$

Zug GR, Chaloupka M, Balazs GH (2006) Age and growth in olive ridley seaturtles (Lepidochelys olivacea) from the north-central Pacific: a skeletochronological analysis. PSZN I: Mar Ecol 27:263-270

Submitted: February 6, 2008; Accepted: May 27, 2008

Proofs received from author(s): July 11, 2008 